\title{
Quantification of intermarket influence on the Australian All Ordinary Index based on optimization techniques
}
C. D. Tilakaratne ${ }^{1}$
S. A. Morris ${ }^{2}$
M. A. Mammadov ${ }^{3}$
C. P. Hurst ${ }^{4}$

(Received 21 July 2006; revised 17 April 2007)

\begin{abstract}
We quantify the influence from the US S\&P 500 Index, along with those from major European and Asian stock market indices, on the Australian All Ordinary Index (AORD). Weights were derived to optimise the average rank correlation between the current day's relative return of the AORD and the weighted sum of the lagged relative returns of the potential influential markets. During the study period, the previous day's Close prices of the considered influential markets had the highest combined influence on the current day's Close price of the AORD. The US S\&P 500 Index contributed most to this combined influence followed by the UK FTSE 100 Index. This study suggests that the use of these two key indices helps predict the current day Close price of the AORD.
\end{abstract}

See http://anziamj.austms.org.au/ojs/index.php/ANZIAMJ/article/view/54 for this article, (c) Austral. Mathematical Soc. 2007. Published May 6, 2007. ISSN $1446-8735$ 


\section{Contents}

1 Introduction

C105

2 Methodology

C106

2.1 Optimization problem .............. . C108

2.2 Rank correlation measure . . . . . . . . . . . C C110

3 Data and numerical experiments $\quad$ C111

3.1 Data and data preprocessing . . . . . . . . . . . . C111

3.2 Numerical experiments . . . . . . . . . . . . . . . C C112

4 Results of numerical experiments $\quad \mathbf{C 1 1 2}$

5 Conclusions $\quad \mathrm{C115}$

$\begin{array}{ll}\text { References } & \text { C116 }\end{array}$

\section{Introduction}

The term intermarket influence, first coined by Murphy [6], is defined as the analysis of relationships between financial markets and their influences on each other [5]. We define Intermarket Influence Analysis as the study of relationships between the current price (or a derivative of price) of a dependent market with the lagged price (or a derivative thereof) of one or more influential markets. Pan et al. [7] revealed that intermarket influence improves the prediction accuracy of stock market prices.

Past studies confirm that most of the the world's major stock markets are integrated $[1,8,9]$. Hence, any stock market should be considered as a part of a single global system. The influence from one stock market on a dependent market includes the influence from one or more stock markets on the former. 
If there is a set of influential markets to a given dependent market, it is not straightforward to separate influence from individual influential markets. Instead, we can measure the strength of the influence from one influential market on the dependent market, compared to the influence from the other influential markets. A better way is to measure the combined influence from the set of influential markets and find the contribution from each influential market to the combined influence.

Currently intermarket influence is an important consideration among investors and decision makers. However, there seems to be a distinct lack of quantification. The article quantifies the intermarket influences from the US S\&P 500 Index (GSPC) and major European and Asian stock market indices on the Australian All Ordinary Index (AORD), which is our target market. Quantification measures may be useful for identifying the market inputs in predictive models.

Results show that the previous day's Close prices of the considered influential markets had the highest combined influence on the current day's Close price of the AORD during the study period: $77 \%$ of this influence was contributed by the US S\&P 500; whereas the contribution from the UK FTSE 100 Index was $16 \%$. Therefore, the current day's Close prices of these two markets help predict the next day's direction (up or down) of the AORD.

Section 2 introduces the optimization problem relating to quantifying intermarket influences and measures of influence. Section 3 describes the data and numerical experiments. Section 4 presents the results of numerical experiments. Section 5 concludes.

\section{$2 \quad$ Methodology}

Quantification of intermarket influences on the target market was carried out by finding the weights which maximise the average rank correlation between 
the current day's relative return of the target market and the weighted sum of the lagged relative returns of potential influential markets, over a number of windows of a fixed size. There is a possibility that the maximum value leads to a conclusion about a relationship which does not exist in reality. In contrast, the average is more conservative in this respect. Therefore, instead of selecting the maximum value of the optimal rank correlation, the average was considered.

The objective function to be minimised (described in Section 2.1) is defined by Spearman's correlation coefficient, which uses ranks of data. Therefore, the objective function is discontinuous. Solving such a global optimization problem is difficult, because of the unavailability of gradients/subgradients. In addition to this difficulty, the objective function is piecewise constant, which makes solving the optimization problem even more difficult. Thus there are two possibilities, either the objective function is discontinuous, or it is differentiable with a gradient of zero; that is, at points where a gradient is computable, it does not help to improve the current point (solution).

A global optimization algorithm was used [3, 4]. It uses a line search mechanism where the descent direction is obtained via a dynamical system approach. The performance of this algorithm has been demonstrated in solving different optimization problems with discontinuous objective functions $[2$, e.g.].

Spearman's rank correlation coefficient was used instead of Pearson's correlation coefficient due to the following reasons.

- Stock market time series are generally non-linear and non-stationary (variance varies with time). Unlike Pearson's correlation coefficient, rank correlation measures (such as Spearman's rank correlation coefficient) assess how well an arbitrary monotonic function describes the relationship between two variables. 
- Rank correlation coefficients are non-parametric measures of correlation. No assumption about frequency distributions of variables is required.

Correlation structure between stock markets change with time [8]. Therefore, rank correlation coefficients were calculated for windows of size 22 days. 22 days of a stock market time series represent a trading month.

\subsection{Optimization problem}

Let $Y(t)$ be the relative return of the Close price of a selected dependent market at time $t$ and $X_{j}(t)$ be the relative return of the Close price of the $j$ th influential market at time $t$. Also let

$$
X_{w}(t-i)=\sum_{j} w_{j} X_{j}(t-i),
$$

where $w_{j} \geq 0, j=1,2, \ldots, m$, are the weights assigned for each influential market $X_{j}, m$ is the total number of influential markets, and $i$ stands for a time lag.

The aim is to find the optimal weights, $w=\left(w_{1}, \ldots, w_{j}\right)$, which maximise the rank correlation between $Y(t)$ and $X_{w}(t-i)$ for a given window and time lag $i$. In the calculations, we consider $i=0,1,2,3,4$, which represent influence within a week. $i=0$ gives the same day correlation between the Close price of the dependent market and a selected combination of the Close prices of influential markets. $i=1$ gives the correlation between the current day's Close price of the dependent market and a combination of previous day's Close prices of influential markets and this correlation is referred as the previous day's combined influence from the influential markets on the dependent markets. Other time lags can be defined in a similar manner. 
Correlation coefficients are calculated over a number of fixed time periods (windows). A window is defined as

$$
T\left(t^{0}, l\right)=\left\{t^{0}, t^{0}+1, \ldots, t^{0}+(l-1)\right\},
$$

where $t^{0}$ is the starting date of the window and $l$ is its size (in days).

For given lag $i$, the correlation between the variables $Y(t)$ and $X_{w}(t-i)$, defined on the window $t \in T\left(t^{0}, l\right)$, is denoted as

$$
C^{i}(w)=\operatorname{Corr}\left(Y(t), X_{w}(t-i) \| T\left(t^{0}, l\right)\right) .
$$

For a particular window $T\left(t^{0}, l\right)$ and time lag $i$, the optimization problem is formulated as

$$
\begin{array}{ll}
\text { Maximise } & C^{i}(w), \\
\text { such that } & \sum_{j} w_{j}=1, \quad w_{j} \geq 0, \quad j=1,2, \ldots, m .
\end{array}
$$

In this way, the optimal weights, $w_{j}, j=1,2, \ldots, m$, are obtained for a given combination of influential markets, on the fixed window $T\left(t^{0}, l\right)$. The optimal correlation changes according to the starting point of the window. To define optimal weights for a long time period, the following method is applied.

Let $[1, T]$ be the whole period. This period is divided into $n$ non-overlapping windows of size $l$ (we assume, without loss of generality, that $T$ is divisible by $l$ ),

$$
T\left(t_{k}, l\right), \quad k=1,2,3, \ldots, n
$$

so that

$$
\begin{aligned}
& T\left(t_{k}, l\right) \cap T\left(t_{k^{\prime}}, l\right)=\phi, \quad \text { for all } k \neq k^{\prime}, \\
& \bigcup_{k=1}^{n} T\left(t_{k}, l\right)=[1, T] .
\end{aligned}
$$


For given $i$, the correlation coefficient on a window $T\left(t_{k}, l\right)$ is denoted as

$$
C_{k}^{i}(w)=\operatorname{Corr}\left(Y(t), X_{w}(t-i) \| T\left(t_{k}, l\right)\right), \quad k=1, \ldots, n .
$$

Instead of maximising correlation over a single window, the average of correlation coefficients over $n$ windows is maximised. We are interested in the strength of the correlation, but not the direction (that is, either positively or negatively correlated). Therefore, the absolute value of the correlation coefficient is considered when calculating the average. Finally, the optimization problem is redefined as

$$
\begin{array}{ll}
\text { Maximise } & f^{i}(w)=\frac{1}{n} \sum_{k=1}^{n}\left|C_{k}^{i}(w)\right|, \\
\text { such that } \quad \sum_{j} w_{j}=1, \quad w_{j} \geq 0, \quad j=1,2, \ldots, m,
\end{array}
$$

where $i=0,1,2,3,4$ is the time lag. In the calculations, we set the window size $l=22$. The above optimization process was repeated by adding the potential influential markets in a stepwise fashion to (1), starting with the GSPC.

\subsection{Rank correlation measure}

Spearman's rank correlation coefficient was used as rank correlation measure in (9). For two variables $X$ and $Y$, Spearman's rank correlation coefficient is

$$
r_{s}=\frac{n\left(n^{2}-1\right)-6 \sum d^{2}-\left(T_{x}+T_{y}\right) / 2}{\sqrt{\left(n\left(n^{2}-1\right)-T_{x}\right)\left(n\left(n^{2}-1\right)-T_{y}\right)}}
$$

where $n$ is the total number of bivariate observations, $d$ is the difference between the rank of $x$ and the rank of $y$, and $T_{x}$ and $T_{y}$ are the number of tied observations of $X$ and $Y$, respectively. 


\section{Data and numerical experiments}

\subsection{Data and data preprocessing}

The Data set consists of the daily Close indices from 26th November 1990 to 30th December 2005 of the Australian All Ordinary Index (AORD) and seven stock markets which were considered to be the potential influential markets of the AORD.

The considered influential markets are the US S\&P 500 Index (GSPC), the UK FTSE 100 Index (FTSE), French CAC 40 Index (FCHI), German DAX Index (GADXI), Hong Kong Hang Seng Index (HSI), Singapore Straits Times Index (STI), and Japanese Nikkei 225 Index (N225). The GSPC is widely considered as the market leader. The FTSE, FCHI, and GDAXI are major stock market indices in Europe, and HSI, STI, and N225 are major Asian stock market indices.

Since different stock markets are closed on different holidays, the regular time series data sets considered have missing values. If no trading took place on a particular day, the rate of change of price should be zero. Therefore, the missing values of the Close price were replaced by the corresponding Close price of the last trading day.

Relative Returns (RR) of the daily Close price of the stock market indices were used for the analysis.

$$
\mathrm{RR}(t)=\frac{P(t)-P(t-1)}{P(t-1)},
$$

where $\operatorname{RR}(t)$ is the relative return and $P(t)$ is the Close price of a selected index on day $t$ respectively. Returns are preferred to price, since returns for different stocks are comparable on an equal basis. 


\subsection{Numerical experiments}

The weights which maximise the average Spearman's rank correlation between the weighted sum of the lagged relative returns of the potential influential markets and the current day's relative return of the AORD were found.

\section{Results of numerical experiments}

This section presents the quantification results. Table 1 shows the average optimal Spearman's correlation between the relative return of the AORD and the weighted sum of the relative returns of the different combinations of influential markets at different time lags. Combinations considered are

$$
\begin{aligned}
& (\mathrm{A}) \equiv \mathrm{GSPC}, \\
& (\mathrm{B}) \equiv(\mathrm{A})+\mathrm{FTSE}, \\
& (\mathrm{C}) \equiv(\mathrm{B})+\mathrm{FCHI}, \\
& (\mathrm{D}) \equiv(\mathrm{C})+\mathrm{GDAXI}, \\
& (\mathrm{E}) \equiv(\mathrm{D})+\mathrm{HSI}, \\
& (\mathrm{F}) \equiv(\mathrm{E})+\mathrm{STI}, \\
& (\mathrm{G}) \equiv(\mathrm{F})+\mathrm{N} 225 .
\end{aligned}
$$

During the study period, the combined influence for each market combination is the highest at time lag 1 and each combination showed an significant influence at time lag 1 (Table 1). The combined influence at lag 1 was increased by $2.19 \%$ when the FTSE was added to the combination which includes the GSPC alone. Thereafter, it remained largely unchanged when the additional stock markets were added. At lag 0, the correlation was significantly high only for the influential market combinations which include at least one Asian market. This value increased by $36.56 \%$ when the HSI was 
TABLE 1: The optimal Spearman's correlation between the AORD relative return and the weighted sum of the relative returns for different combinations of influential markets at different time lags. Percentage increase of optimal correlation when each market was added is also shown. * denotes the correlations which are significant (one sided) at 5\% level. Time lag 0 represents the correlation.

\begin{tabular}{cccccccc}
\hline $\begin{array}{c}\text { Time } \\
\text { Lag }\end{array}$ & $(\mathrm{A})$ & $(\mathrm{B})$ & $(\mathrm{C})$ & $(\mathrm{D})$ & $(\mathrm{E})$ & $(\mathrm{F})$ & $(\mathrm{G})$ \\
\hline 0 & 0.183 & 0.253 & 0.255 & 0.279 & $0.381^{*}$ & $0.403^{*}$ & $0.429^{*}$ \\
& & $38.25 \%$ & $0.79 \%$ & $9.41 \%$ & $36.56 \%$ & $5.77 \%$ & $6.45 \%$ \\
1 & $0.456^{*}$ & $0.466^{*}$ & $0.470^{*}$ & $0.470^{*}$ & $0.470^{*}$ & $0.471^{*}$ & $0.470^{*}$ \\
& & $2.19 \%$ & $0.85 \%$ & $0.00 \%$ & $0.00 \%$ & $0.21 \%$ & $0.00 \%$ \\
2 & 0.185 & 0.185 & 0.187 & 0.187 & 0.194 & 0.194 & 0.194 \\
& & $0.00 \%$ & $1.08 \%$ & $0.00 \%$ & $3.74 \%$ & $0.00 \%$ & $0.00 \%$ \\
3 & 0.175 & 0.184 & 0.184 & 0.185 & 0.192 & 0.192 & 0.193 \\
& & $5.14 \%$ & $0.00 \%$ & $0.54 \%$ & $3.78 \%$ & $0.00 \%$ & $0.52 \%$ \\
4 & 0.157 & 0.170 & 0.174 & 0.176 & 0.180 & 0.188 & 0.188 \\
& & $8.28 \%$ & $2.35 \%$ & $1.15 \%$ & $2.27 \%$ & $4.44 \%$ & $0.00 \%$ \\
\hline
\end{tabular}


TABLE 2: The weights for the influential markets considered at different time lags. The weights which optimize Spearman's rank correlation between the AORD relative returns and weighted sum of relative returns of influential market combination $(\mathrm{G})$. Time lag 0 represents the correlation.

\begin{tabular}{cccccccc}
\hline $\begin{array}{c}\text { Time } \\
\text { Lag }\end{array}$ & GSPC & FTSE & FCHI & GDAXI & HSI & STI & N225 \\
\hline 0 & 0.015 & 0.112 & 0.037 & 0.031 & 0.347 & 0.156 & 0.301 \\
1 & 0.772 & 0.156 & 0.066 & 0.006 & 0.000 & 0.000 & 0.000 \\
2 & 0.487 & 0.011 & 0.000 & 0.000 & 0.502 & 0.000 & 0.000 \\
3 & 0.330 & 0.001 & 0.000 & 0.003 & 0.332 & 0.251 & 0.081 \\
4 & 0.325 & 0.012 & 0.001 & 0.004 & 0.333 & 0.324 & 0.000 \\
\hline
\end{tabular}

added to the market combination of the us and the European markets, and this influence kept on increasing when other Asian markets were added. No combined influence of any combination of markets at time lags greater than one are significant.

The weight corresponding to an influential market quantifies the relative strength of the influence from this market on the AORD. Therefore, the weights measure the contribution from corresponding influential markets to the combined influence. For this purpose it is sufficient to consider the influential market combination $(\mathrm{G})$, since it covers all potential influential markets considered. Table 2 presents the weights for the influential markets considered at different time lags.

During the study period, correlations between the Close price of the AORD and the same day Close prices of the Asian stock markets, particularly HSI (0.347) and N225 (0.301), were stronger than those of the European markets and the GSPC (Table 2). The GSPC showed the lowest contribution to the correlation. 
The previous day's Close price of the GSPC had a very strong influence (0.772) on the current day Close price of the AORD. This influence was stronger than the influence from any other market. The contribution from the previous day's Close price of the FTSE was 15.6\%. The influence from the previous day's Close price of the Asian markets on the current day Close price of the AORD was negligible. Although some weights at time lags greater than one suggests a contribution, the corresponding combined influences are not significant (Table 1). Therefore, the influence from the Close prices of two or more days in the past of any influential market on the current day Close price of the AORD was negligible.

The important feature of Table 2 is that the GSPC has the minimum contribution at time lag zero and the maximum contribution at time lag one. This implies that the quantification was successful.

\section{Conclusions}

The conclusions derived from the analysis are summarised.

1. In addition to the previous day's Close price of the GSPC, that of the FTSE also influenced the current day's Close price of the AORD during the study period. The contribution from the GSPC was $77 \%$ and that from the FTSE was $16 \%$. However, the influences of all other markets considered were negligible.

2. The Close price of the Australian all Ordinary Index was highly correlated with same day Close prices of the major Asian markets, particularly HSI and N225 during the study period.

3. The Close prices of two or more days in the past of all the considered markets did not show a significant influence on the Australian All Ordinary Index. 
The previous day's Close price of the US S\&P 500 Index, together with that of the UK FTSE 100 Index, help predict the next day's Close price of the Australian All Ordinary Index. The weights derived in this study are useful for identifying the market inputs in predictive models. The optimization technique proposed in this study is suitable to quantify the influence from a selected set of potential influential markets on a given target market.

Acknowledgements Thanks go to Dr H. Pan and Associate Professor J. Yearwood for their advice at the beginning of this study.

\section{References}

[1] Eun, C. S., and Shim, S. (1989). International Transmission of Stock Market Movements. The Journal of Financial and Quantitative Analysis, 24(2), 241-256. C105

[2] Koubor, S., Ugon, J., Mammadov, M. A., Rubinov A. M., and Kruger, A. (2006). Coverage in WLAN: Optimization Model and Algorithm, Proceeding of the First IEEE International Conference on Wireless Broadband and Ultra Wideband Communications, Sydney, Australia. C107

[3] Mammadov, M. A. (2004). A new global optimization algorithm based on dynamical systems approach. In A. Rubinov and M. Sniedovich (Editors), Proceedings of The Sixth International Conference on Optimization: Techniques and Applications (ICOTA6), Ballarat, Australia. C107

[4] Mammadov, M. A., Rubinov, A. M., and Yearwood, J. (2005).

Dynamical systems described by relational elasticities with applications to global optimization, In V. Jeyakumar and A. Rubinov (Eds.) 
Continuous Optimisation: Current Trends and Applications, Springer, 365-387. C107

[5] Mendelsohn, L. B. (2000). Trend Forecasting with Technical Analysis. Market Technologies Corporation. C105

[6] Murphy, J. J. (1991). Intermarket Technical Analysis. John Wiley and Sons. C105

[7] Pan, H., Tilakaratne, C., and Yearwood, J. (2005). Predicting the Australian Stock Market Index Using Neural networks Exploiting Dynamical Swings and Intermarket Influences. Journal of Research and Practice in Information Technology, 37(1), 43-55.

https://www.acs.org.au/jrpit/JRPIT_Volumes.html C105

[8] Wu, C., and Su, Y. (1998). Dynamic Relations among International Stock Markets. International Review of Eeconomic and Finance, 7(1), 63-84. C105, C108

[9] Yang, J., Khan, M. M., and Pointer, L. (2003). Increasing Integration Between the United States and Other International Stock Markets?; A Recursive Cointegration Analysis. Emerging Markets Finance and Trade, 39(6), 39-53. C105 


\section{Author addresses}

1. C. D. Tilakaratne, Center for Informatics and Applied Optimization, School of Information Technology and Mathematical Sciences, University of Ballarat, PO Box 663, Ballarat, Victoria 3353, Australia.

mailto:ctilakaratne@students.ballarat.edu.au

2. S. A. Morris, Center for Informatics and Applied Optimization, School of Information Technology and Mathematical Sciences, University of Ballarat, PO Box 663, Ballarat, Victoria 3353, Australia.

3. M. A. Mammadov, Center for Informatics and Applied Optimization, School of Information Technology and Mathematical Sciences, University of Ballarat, PO Box 663, Ballarat, Victoria 3353, Australia.

4. C. P. Hurst, Center for Informatics and Applied Optimization, School of Information Technology and Mathematical Sciences, University of Ballarat, PO Box 663, Ballarat, Victoria 3353, Australia. 